\section{TATRA \\ MOUNTaiNS \\ Mathematical Publications}

DOI: $10.2478 /$ tmmp-2013-0017

Tatra Mt. Math. Publ. 55 (2013), 37-45

\title{
ON CERTAIN APPROXIMATION PROBLEM CONNECTED WITH THE SUMS OF SUBSERIES
}

\author{
Roman Witula - Konrad KaczmareK - Edyta HetmanioK - \\ - DAmian SŁota
}

\begin{abstract}
In this paper a problem of approximating the real numbers by using the series of real numbers is considered. It is proven that if the given family of sequences of real numbers satisfies some conditions of set-theoretical nature, like being closed under initial subsequences and (additionally) possessing properties of adding and removing elements, then it automatically possesses some approximating properties, like, for example, reaching supremum of the set of sums of subseries.
\end{abstract}

\section{Introduction}

Problem, discussed in this paper, concerns the approximation of the real numbers by using the series of real numbers. It turns out that if the class of infinite sequences of real numbers satisfies some simple set relations (like being closed under initial subsequences, properties of adding and removing elements), then the approximation of a real number by using the appropriate series results immediately from the nature of real numbers. Authors of the well-known and widely read monographs [1]-4] usually omit this problem. However, we have found it interesting, therefore we decided to devote the current paper to this issue. We hope that the obtained results may be worthy of our interest. Let us emphasize that the case of real sequences is definitely easier than the case of multidimensional sequences (starting with a dimension of $n=2$ ). The infinitely dimensional case is almost completely out of discussion, since it is not easy to consider and requires another kind of assumptions.

Let

$$
\mathfrak{s}:=\left\{\left\{a_{n}\right\}_{n=1}^{\infty}: a_{n} \geqslant 0, n \in \mathbb{N} \text { and } \sum_{n=1}^{\infty} a_{n}<\infty\right\} .
$$

(C) 2013 Mathematical Institute, Slovak Academy of Sciences.

2010 Mathematics Subject Classification: 40A05, 40A25.

Keywords: approximation by sums of subseries, property of sets closed under initial subsequences, properties of adding and removing elements. 
Definition 1. We say that a nonempty set $w \subset \mathfrak{s}$ is closed under initial subsequences (abbreviated to cuis-set, for shortness of notation) if from the fact that for a given sequence $\left\{a_{n}\right\}_{n=1}^{\infty} \in \mathfrak{s}$ and for any $N \in \mathbb{N}$ there exists $\left\{b_{n}\right\}_{n=1}^{\infty} \in w$ such that $a_{n}=b_{n}$ for $n=1,2, \ldots, N$, it follows that $\left\{a_{n}\right\}_{n=1}^{\infty} \in w$.

Let $\left\{a_{n}\right\}_{n=1}^{\infty} \in \mathfrak{s}$ and $x \in \mathbb{R}_{+}$. Let us also fix the nonempty set $w \subset \mathfrak{s}$. Now, suppose that for every $\varepsilon>0$ there exists a subsequence $\left\{a_{n_{i}}\right\}_{i=1}^{\infty} \in w$ such that

$$
x-\varepsilon<\sum a_{n_{i}}<x .
$$

We prove that if additionally $w$ is closed under initial subsequences, then there exists a subsequence $\left\{a_{n_{i}}\right\}_{i=1}^{\infty} \in w$ such that $\sum a_{n_{i}}=x$.

Moreover, many different examples of families $w \subset \mathfrak{s}$ which are closed under initial subsequences will be presented. The rôle of condition "closed under initial subsequences" will also be discussed.

\section{Main results}

TheOREm 2. Let $w \subset \mathfrak{s}$ be a nonempty set which is closed under initial subsequences. Let $\left\{a_{n}\right\} \in \mathfrak{s}$ and $x \in \mathbb{R}_{+}$. If for every $\varepsilon>0$ there exists a subsequence $\left\{a_{n_{i}}\right\} \in w$ such that

$$
x-\varepsilon<\sum a_{n_{i}}<x
$$

then there also exists a subsequence $\left\{a_{n_{i}}\right\} \in w$ such that $\sum a_{n_{i}}=x$.

P r o o f. Let us set

$$
\begin{gathered}
k_{1}:=\min \left\{k \in \mathbb{N}: k>1 \text { and } \sum_{n \geqslant k} a_{n}<x\right\}, \\
\varepsilon_{1}:=x-\sum_{n \geqslant k_{1}} a_{n} .
\end{gathered}
$$

Certainly, each subsequence $\left\{a_{n_{i}}\right\}$, such that $x-\varepsilon_{1}<\sum a_{n_{i}}<x$, must include some subsequence of the sequence $\left\{a_{1}, \ldots, a_{k_{1}-1}\right\}$. Since there is finitely many of all subsequences of the sequence $\left\{a_{1}, \ldots, a_{k_{1}-1}\right\}$, therefore, with regard to the assumption (11), a subsequence $\left\{a_{n_{i}^{\prime}}\right\}_{i=1}^{s_{1}}$ of the sequence $\left\{a_{i}\right\}_{i=1}^{k_{1}-1}$ exists and the condition

for every $\varepsilon>0$ there exists a subsequence $\left\{a_{n_{i}}\right\} \in w$ such that $x-\varepsilon<\sum a_{n_{i}}<x$ and the $s_{1}$ initial elements

of which are $a_{n_{1}^{\prime}}, \ldots, a_{n_{s_{1}}^{\prime}}$

is satisfied. 


\section{ON CERTAIN APPROXIMATION PROBLEM}

Let us set $x_{1}=x-\left(a_{n_{1}^{\prime}}+\cdots+a_{n_{s_{1}}^{\prime}}\right)$. We put

$$
\begin{gathered}
k_{2}:=\min \left\{k \in \mathbb{N}: k>k_{1} \text { and } \sum_{n \geqslant k} a_{n}<x_{1}\right\}, \\
\varepsilon_{2}:=x_{1}-\sum_{n \geqslant k_{2}} a_{n} .
\end{gathered}
$$

Each subsequence $\left\{a_{n_{i}}\right\} \in w$, such that $x-\varepsilon_{2}<\sum a_{n_{i}}<x$ and the $s_{1}$ initial elements of which are $a_{n_{1}^{\prime}}, \ldots, a_{n_{s_{1}}^{\prime}}$, must include some subsequence of the sequence $\left\{a_{k_{1}}, \ldots, a_{k_{2}-1}\right\}$. From this and from (2), it results that there exists a subsequence $\left\{a_{n_{i}^{\prime}}\right\}_{i=s_{1}+1}^{s_{2}}$ of the sequence $\left\{a_{i}\right\}_{i=k_{1}}^{k_{2}-1}$ satisfying the condition

for every $\varepsilon>0$ there exists a subsequence $\left\{a_{n_{i}}\right\} \in w$ such that $x-\varepsilon<\sum a_{n_{i}}<x$ and the $s_{2}$ initial elements

of which are $a_{n_{1}^{\prime}}, \ldots, a_{n_{s_{2}}^{\prime}}$.

Let us set $x_{2}:=x_{1}-\left(a_{n_{s_{1}+1}^{\prime}}+\cdots+a_{n_{s_{2}}^{\prime}}\right)$. We put $k_{3}:=\min \left\{k \in \mathbb{N}: k>k_{2}\right.$ and $\left.\sum_{n \geqslant k} a_{n}<x_{2}\right\}, \varepsilon_{3}:=x_{2}-\sum_{n \geqslant k_{3}} a_{n}$. Each subsequence $\left\{a_{n_{i}}\right\} \in w$, such that $x-\varepsilon_{3}<\sum a_{n_{i}}<x$ and the $s_{2}$ initial elements of which are $a_{n_{1}^{\prime}}, \ldots, a_{n_{s_{2}}^{\prime}}$, must include some subsequence of the sequence $\left\{a_{k_{2}}, \ldots, a_{k_{3}-1}\right\}$. This, together with (3), implies that there exists a subsequence $\left\{a_{n_{i}^{\prime}}\right\}_{i=s_{2}+1}^{s_{3}}$ of the sequence $\left\{a_{n_{i}}\right\}_{i=k_{2}}^{k_{3}-1}$ such that for each $\varepsilon>0$ there exists a subsequence $\left\{a_{n_{i}}\right\} \in w$ with the property $x-\varepsilon<\sum a_{n_{i}}<x$ and the $s_{3}$ initial elements of which are $a_{n_{1}^{\prime}}, \ldots, a_{n_{s_{3}}^{\prime}}$, etc.

The above inductive process leads to the definition of infinite subsequence $\left\{a_{n_{i}^{\prime}}\right\}$. In accordance with property of closed under initial subsequences, the sequence $\left\{a_{n_{i}^{\prime}}\right\} \in w$. Moreover, we will show that $\sum a_{n_{i}^{\prime}}=x$. For this purpose we suppose that $\sum a_{n_{i}^{\prime}}<x$.

Let $\varepsilon_{0}:=x-\sum a_{n_{i}^{\prime}}$. Let us denote

$$
k_{0}:=\min \left\{k \in \mathbb{N}: \sum_{n \geqslant k} a_{n}<\frac{\varepsilon_{0}}{2}\right\} .
$$

Definition of subsequence $\left\{a_{n_{i}^{\prime}}\right\}$ implies that for each $\varepsilon>0$ there exists a subsequence $\left\{a_{n_{i}}\right\} \in w$ such that

$$
x-\varepsilon<\sum a_{n_{i}}<x
$$

and $a_{n_{1}^{\prime}}, \ldots, a_{n_{k_{0}}^{\prime}}$ form the initial $k_{0}$ elements of subsequence $\left\{a_{n_{i}}\right\}$. In the sequel, there exists a subsequence $\left\{a_{n_{i}}\right\} \in w$ such that

$$
x-\frac{\varepsilon_{0}}{2}<\sum a_{n_{i}}<x
$$


and the $k_{0}$ initial elements of which are $a_{n_{1}^{\prime}}, \ldots, a_{n_{k_{0}}^{\prime}}$. From the last property and from the previous assumptions, we get

$\sum a_{n_{i}}=a_{n_{1}^{\prime}}+\cdots+a_{n_{k_{0}}^{\prime}}+\sum_{i>k_{0}} a_{n_{i}} \leqslant \sum a_{n_{i}^{\prime}}+\sum_{n \geqslant k_{0}} a_{n}<\sum a_{n_{i}^{\prime}}+\frac{\varepsilon_{0}}{2}=x-\frac{\varepsilon_{0}}{2}$,

in contradiction with (41).

Corollary 3. Let $a_{n}>0, n \geqslant 1, \lim a_{n}=0, w \subset \mathfrak{s}$ and let $w$ be a cuis-set. If $\sup \left\{\sum a_{n_{i}}:\left\{a_{n_{i}}\right\} \in w\right\}<\infty$, then there exists a subsequence $\left\{a_{n_{i}^{\prime}}\right\} \in w$ such that $\sum a_{n_{i}^{\prime}}=\sup \left\{\sum a_{n_{i}}:\left\{a_{n_{i}}\right\} \in w\right\}$.

EXAMPLE 4 . We now present the examples of five families $w \subset \mathfrak{s}$ closed under initial subsequences:

$$
\begin{aligned}
w_{1}= & \left\{\left\{a_{n}\right\} \in \mathfrak{s}:\left\{a_{n}\right\} \subset \mathbb{Q}\right\}, \\
w_{2}= & \left\{\left\{a_{n}\right\} \in \mathfrak{s}: a_{n} \geqslant a_{n+1}, n \in \mathbb{N}\right\}, \\
w_{3}= & \left\{\left\{a_{n}\right\} \in \mathfrak{s}: \text { decimal expansion of number } a_{n}\right. \text { is formed } \\
& \text { only by zeros and ones }\},
\end{aligned}
$$

for some fixed $\left\{a_{n}\right\} \in \mathfrak{s}$, the family $w_{4}$ is created from all infinite

subsequences of $\left\{a_{n}\right\}$,

$$
w_{5}=\left\{\left\{a_{n}\right\} \in \mathfrak{s}: a_{2 n-1} \leqslant a_{2 n} \text { and } a_{2 n} \geqslant a_{2 n+1}, n \geqslant 1\right\} .
$$

Remark 5 (about the meaning of cuis-set condition in Theorem 2). Let

$$
b_{n}=: \begin{cases}9 \cdot 10^{-(n+1) / 2}, & n \in 2 \mathbb{N}-1, \\ 10^{-n / 2}, & n \in 2 \mathbb{N} .\end{cases}
$$

If we describe a set $w \subset \mathfrak{s}$ in the following way: $\left\{a_{n}\right\} \in w \Leftrightarrow\left\{a_{n}\right\} \in \mathfrak{s}$ and $\sum d\left(a_{n}\right)<\infty$ where $d: \mathbb{R} \rightarrow \widetilde{\mathbb{R}}$ and, by definition, $d(x)$ denotes the minimal number of nines which must appear in decimal expansion of number $x$, then one can easily notice that $w$ is not a cuis-set. Simultaneously, for every $\varepsilon>0$ there exists a subsequence $\left\{b_{n_{i}}\right\} \in w$ such that $1-\varepsilon<\sum b_{n_{i}}<1$ and there does not exist a subsequence $\left\{b_{n_{i}}\right\} \in w$ such that $\sum b_{n_{i}}=1$.

The next example constitutes a supplement of Corollary 3 .

EXAMPLE 6 . We give the examples of sequences $\left\{a_{n}\right\}$, such that $\sum a_{n}=\infty$, satisfying the condition: for every subsequence $\left\{a_{n_{i}}\right\}$ if $\left\{a_{n_{i}}\right\} \in w$, then $\sum a_{n_{i}}<+\infty$, where $w$ is one of the following three sets:

a) $\left\{\left\{b_{n}\right\} \subset \mathbb{R}_{+}: b_{n} \geqslant b_{n+1}, n \in \mathbb{N}\right\}$,

b) $\left\{\left\{b_{n}\right\} \subset \mathbb{R}_{+}: b_{2 n-1} \leqslant b_{2 n}\right.$ and $\left.b_{2 n} \geqslant b_{2 n+1}, n \geqslant 1\right\}$,

c) $\left\{\left\{b_{n}\right\} \subset \mathbb{R}_{+}: b_{2 n+1} \geqslant b_{2 n}, n \in \mathbb{N}\right\}$. 


\section{ON CERTAIN APPROXIMATION PROBLEM}

Definitions of the respective sequence $\left\{a_{n}\right\}$ :

a) We take $a_{1}=1$. If elements $a_{1}, \ldots, a_{s}$ and $a_{s}=n^{-2}$ are already given, then we put

$$
a_{s+i}=\frac{1}{(n+2)^{2}}+\frac{i}{(n+2)}\left(\frac{1}{(n+1)^{2}}-\frac{1}{(n+2)^{2}}\right) \quad \text { for } \quad 1 \leqslant i \leqslant n+2 .
$$

It is obvious that the last defined element $a_{s+n+2}$ is equal to $\frac{1}{(n+1)^{2}}$.

b) All we need here is to take

$$
a_{2^{n}+i}:=2^{-n}-i 2^{-2 n-1} \quad \text { for } \quad 0 \leqslant i \leqslant 2^{n}, i \neq 2^{n}-1, n \in \mathbb{N}_{0}
$$

and

$$
\begin{aligned}
& a_{2^{n}-1}:=a_{2^{n}+1}, \quad n \in \mathbb{N}_{0} . \\
& \text { c) } \quad a_{n}:= \begin{cases}(n-1)^{-1}, & n=k^{2}+1, \quad k \in \mathbb{N}, \\
n^{-1}, & \text { for other } n \in \mathbb{N} .\end{cases}
\end{aligned}
$$

Let us notice that in each of these examples, as resulting from Corollary 3 , the appropriate $\sup \left\{\sum a_{n_{i}}:\left\{a_{n_{i}}\right\} \in w\right\}$ is reached, and, which is more (with regard to the form of construction of the sequence $\left\{a_{n}\right\}$ ), the appropriate subsequence $\left\{a_{n_{i}}\right\}$ realizing this supremum can be easily described explicitly.

EXAMPLE 7. Let $w=\left\{\left\{k_{i}^{-1}\right\}_{i=1}^{\infty} \in \mathfrak{s}:\left\{k_{i}\right\}_{i=1}^{\infty}\right.$ be an increasing sequence of positive integers $\}$. Then, $w$ is a cuis-set and simultaneously,

$$
\sup \left\{\sum_{i=1}^{\infty} k_{i}^{-1}:\left\{k_{i}\right\} \in w\right\}=\infty .
$$

The last two examples, more precisely-Corollary [3, and more practically-a desire of eliminating the additional assumption sup $\left\{\sum a_{n_{i}}:\left\{a_{n_{i}}\right\} \in w\right\}<\infty$ from this conclusion (it means, replacing it by the assumptions of set-theoretical nature which will be conditions 1) and 2) from the theorem given below), led us to create our second main result.

Theorem 8. Let $w \subset \mathfrak{s}$. Assume that the set $w$ is a cuis-set and it possesses the following two properties:

1) (Property of adding elements.) If for any $\left\{a_{n}\right\} \in w$ and $k \in \mathbb{N}$ there exists $\omega(k) \in \mathbb{N}, \omega(k) \geqslant k$, such that if $\left\{b_{n}\right\} \in w$ and $\max \left\{b_{n}\right\} \leqslant \min \left\{a_{1}, \ldots, a_{\omega(k)}\right\}$, then the sequence $\left\{c_{n}\right\}$ defined below

$$
c_{n}= \begin{cases}a_{n}, & 1 \leqslant n \leqslant \omega(k), \\ b_{n-\omega(k)}, & n>\omega(k),\end{cases}
$$

belongs to $w$. 
2) (Property of removing elements.) For every $k \in \mathbb{N}$ there exists $k^{\prime} \in \mathbb{N}, k^{\prime} \geqslant k$, such that if $\left\{a_{n}\right\} \in w$, then there exists $s: k^{\prime} \geqslant s \geqslant k$ with the property $\left\{a_{n}\right\}_{n \geqslant s} \in w$.

Let us fix $\left\{a_{n}\right\}$, such that $a_{n}>0, n \geqslant 1, \lim a_{n}=0$. If there exists a subsequence $\left\{a_{n_{i}^{\prime}}\right\} \in w$, then there exists a subsequence $\left\{a_{n_{i}}\right\} \in w$ such that $\sum a_{n_{i}}=\sup \left\{\sum a_{n_{i}^{\prime}}:\left\{a_{n_{i}^{\prime}}\right\} \in w\right\}$.

P r o of. In view of Corollary 3, it suffices to prove that

$$
\sup \left\{\sum a_{n_{i}^{\prime}}:\left\{a_{n_{i}^{\prime}}\right\} \in w\right\}<+\infty .
$$

For this purpose, we assume, on the contrary, that

$$
\sup \left\{\sum a_{n_{i}^{\prime}}:\left\{a_{n_{i}^{\prime}}\right\} \in w\right\}=\infty \text {. }
$$

We will show that then there exists a subsequence $\left\{a_{n_{i}}\right\} \in w$ such that $\sum a_{n_{i}}=$ $+\infty$. The subsequence $\left\{a_{n_{i}}\right\}$ can be constructed on the basis of the following property resulting from properties 1), 2) and the assumption that $w$ is a cuis-set:

if $\left\{a_{n_{i}}\right\} \in w$, then for each $k \in \mathbb{N}$ and $M>0$ there exists a subsequence $\left\{a_{n_{i}^{\prime}}\right\} \in w$ such that $a_{n_{i}^{\prime}}=a_{n_{i}}, 1 \leqslant i \leqslant k$ and $\sum a_{n_{i}^{\prime}}>M$.

Proof of Property (6). Let us fix $\left\{a_{n_{i}}\right\} \in w, k \in \mathbb{N}$ and $M>0$. Let $a_{n} \leqslant$ $\varrho, n \geqslant 1$. We select $t \in \mathbb{N}$ such that

$$
\max \left\{a_{n}\right\}_{n \geqslant t} \leqslant \min \left\{a_{n_{1}}, \ldots, a_{n_{\omega(k)}}\right\},
$$

where $\omega(k)$ is as in the property 1$)$ for the sequence $\left\{a_{n_{i}}\right\}$. Let us put

$$
\delta:=\max \left\{n_{\omega(k)}+1, t\right\} .
$$

Condition (5) implies that there exists a subsequence $\left\{a_{n_{i}^{\prime}}\right\} \in w$ such that $\sum a_{n_{i}^{\prime}}>M+\varrho \delta^{\prime}$, where $\delta^{\prime}$ is the number from property 2) corresponding with number $\delta$. According to the property 2), there exists an index $s: \delta^{\prime} \geqslant s \geqslant \delta$ such that the sequence $\left\{a_{n_{i}^{\prime}}\right\}_{i \geqslant s} \in w$. We get

$$
\sum_{i \geqslant s} a_{n_{i}^{\prime}}=\sum_{i \geqslant 1} a_{n_{i}^{\prime}}-\sum_{i<s} a_{n_{i}^{\prime}}>M+\varrho \delta^{\prime}-\sum_{i<s} a_{n_{i}^{\prime}}>M+\varrho \delta^{\prime}-\varrho s>M .
$$

Simultaneously, we have $\max \left\{a_{n_{i}^{\prime}}\right\}_{i \geqslant s} \leqslant \max \left\{a_{n}\right\}_{n \geqslant t}$ from which, in view of (7) and the property 1 ), we obtain that the sequence $\left\{c_{i}\right\}$ defined as follows

$$
c_{i}=\left\{\begin{array}{lc}
a_{n_{i}}, & 1 \leqslant i \leqslant \omega(k), \\
a_{n_{[i-\omega(k)-1+s]}^{\prime},}, & i>\omega(k),
\end{array}\right.
$$

belongs to $w$ which completes the proof of property (6). 


\section{ON CERTAIN APPROXIMATION PROBLEM}

Now, let us return to the proof of Theorem 8 ,

From (5) it results that there exists a subsequence $\left\{a_{n_{i}^{(1)}}\right\} \in w$ such that $\sum a_{n_{i}^{(1)}}>2$. Let $k_{1}=\min \left\{k \in \mathbb{N}: \sum_{i=1}^{k} a_{n_{i}^{(1)}}>1\right\}$. In general, let us suppose that we have already defined the number $k_{s} \in \mathbb{N}$. From (6) we receive that there exists a subsequence $\left\{a_{n_{i}^{(s+1)}}\right\} \in w$ such that

$$
\begin{gathered}
a_{n_{i}^{(s+1)}}=a_{n_{i}^{(s)}}, \quad 1 \leqslant i \leqslant k_{s}, \\
\sum a_{n_{i}^{(s+1)}}>\sum_{i=1}^{k_{s}} a_{n_{i}^{(s)}}+2 .
\end{gathered}
$$

We take

$$
k_{s+1}:=\min \left\{k \in \mathbb{N}: \sum_{i=1}^{k} a_{n_{i}^{(s+1)}}>1+\sum_{i=1}^{k_{s}} a_{n_{i}^{(s)}}\right\} .
$$

Obviously, $k_{s+1}>k_{s}$. that

The above described algorithm leads us to create the subsequence $\left\{a_{n_{i}}\right\}$ such

$$
a_{n_{i}}=a_{n_{i}^{(s)}}, \quad s \in \mathbb{N}, \quad 1 \leqslant i \leqslant k_{s} .
$$

Since each subsequence $\left\{a_{n_{i}^{(s)}}\right\}_{i \geqslant 1}$ is included in $w$ thus, from the property of closed under initial subsequences, we get that $\left\{a_{n_{i}}\right\} \in w$. Furthermore,

$$
\sum a_{n_{i}} \geqslant \sum_{i=1}^{k_{s}} a_{n_{i}^{(s)}} \geqslant s-1+\sum_{i=1}^{k_{s}} a_{n_{i}^{(1)}}, \quad s \in \mathbb{N},
$$

from which we obtain $\sum a_{n_{i}}=+\infty$; this is contradictory to the assumption saying that for each subsequence $\left\{a_{n_{i}^{\prime}}\right\} \in w$ we have $\sum a_{n_{i}^{\prime}}<+\infty$. In this way, we have shown that the assumption (5) is false, that is

$$
\sup \left\{\sum a_{n_{i}^{\prime}}:\left\{a_{n_{i}^{\prime}}\right\} \in w\right\}<+\infty \text {. }
$$

Remark 9. All the examples of families $w_{k} \subset \mathfrak{s}, k=1, \ldots, 5$, presented after Corollary 3, satisfy the property of being closed under initial subsequences and, simultaneously, the assumptions 1) and 2) from Theorem 8 .

At the end of the paper, we give an example of the family $w$ which is closed under initial subsequences and which possesses none of conditions 1) and 2) from Theorem 8. Moreover, for this family $w$, Theorems 2 and 8 hold true, however, for Theorem 2, in the purely logic sense only. 
EXAMPLE 10. Let us set

$$
w=\left\{\left\{k^{-x}\right\}_{k=1}^{\infty}: x>1\right\} .
$$

The family $w$ is a cuis-set since if $\left\{a_{n}\right\} \in \mathfrak{s}$ and $a_{1}=1$ and $a_{2}=2^{-x}=2^{-y}$ for some $x, y>1$, then $x=y$ and, in consequence, if $a_{1}, a_{2}, \ldots, a_{N}$ are initial elements of some sequence $\left\{b_{n}\right\} \in w$ for every $N \in \mathbb{N}$, then $\left\{a_{n}\right\}=\left\{k^{-x}\right\}_{k=1}^{\infty}$ for some $x>1$. It is very easy to show that conditions 1) and 2) of Theorem 8 do not hold for family $w$.

Simultaneously, let us notice that if for some sequence $\left\{a_{n}\right\} \in \mathfrak{s}$ and $x \in \mathbb{R}_{+}$ the condition (11) would be held, then the sequence $\left\{a_{n}\right\}$ would contain infinitely many of sequences $\left\{k^{-p_{n}}\right\}_{k=1}^{\infty}$, such that $p_{1}>p_{2}>\ldots$ and

$$
\lim _{n \rightarrow \infty} \sum_{k=1}^{\infty} k^{-p_{n}}=x
$$

which would contradict the convergence of series $\sum a_{n}$. Therefore, Theorem 2 holds in the purely logic sense only (we note that the replacement of condition (11) with a similar one but having weak inequalities makes no big change).

Theorem 8 is a different case. Let us fix any sequence $\left\{a_{n}\right\}$

$$
a_{n}>0, \lim a_{n}=0,
$$

including at least one subsequence

$$
\left\{a_{n_{i}^{\prime}}\right\}=\left\{k^{-x_{0}}\right\}_{k=1}^{\infty}, \quad \text { where } x_{0}>1
$$

(obviously, there exist continuum examples of that kind). Since $\lim a_{n}=0$, thus there exist at most finitely many subsequences of the sequence $\left\{a_{n}\right\}$ having the form $\left\{k^{-x}\right\}_{k=1}^{\infty}$ with $x \leqslant x_{0}$, because the sequence $\left\{a_{n}\right\}$ can contain only finitely many numbers $2^{-x}$ with $x \leqslant x_{0}$, since $\sum a_{n}<\infty$. Let us fix the possibly smallest value $x^{*} \leqslant x_{0}$. Then,

$$
\sum_{k=1}^{\infty} k^{-x^{*}}=\sup \left\{\sum_{k=1}^{\infty} k^{-x}: x>1 \text { and }\left\{k^{-x}\right\}_{k=1}^{\infty} \text { is a subsequence of }\left\{a_{n}\right\}\right\},
$$

which proves that the thesis of Theorem 8 is true.

Moreover, let us notice that similar properties as possessed by the above family $w$ are possessed by each of the following sets

$$
\omega_{k}:=\left\{\left\{n^{-x}\right\}_{n=k}^{\infty}: x>1\right\}, \quad \text { where } k=1,2, \ldots
$$

\section{REFERENCES}

[1] LAURENT, P.-J.: Approximation et Optimisation, in: Enseignement des Sciences, Vol. 13, Hermann, Paris, 1972. (In French)

[2] DEvore, R. A.-LOREnTZ, G. G.: Constructive Approximation, in: Grundlehren der Mathematischen Wissenschaften, Vol. 303, Springer-Verlag, Berlin, 1993. 


\section{ON CERTAIN APPROXIMATION PROBLEM}

[3] ACHIEZSER, N. I.: Theory of Approximation, Polish Scientific Publishers, Warsaw, 1957. (In Polish)

[4] TIKHOMIROV, V. M.: Some Questions in Approximation Theory. Moscow University Press, Moscow, 1976. (In Russian)

Received October 19, 2012

Institute of Mathematics

Silesian University of Technology

Kaszubska 23

$P L-44-100$ Gliwice

POLAND

E-mail: roman.witula@polsl.pl konrad.kaczmarek@polsl.pl edyta.hetmaniok@polsl.pl

damian.slota@polsl.pl 